\title{
Island Universes: Galactic General Relativity without Dark Matter
}

\author{
Günter Scharf \\ Physics Institute, University of Zürich, Winterthurerstr. 190 , CH-8057 Zürich, Switzerland \\ Email: scharf@physik.uzh.ch
}

\begin{abstract}
We apply the perturbative technique which was previously developed in cosmology to now describe the gravitational field of a galaxy with arbitrary rotation curve. The rotation curve fixes the background metric. We use a Schwarzschild background as starting point, however with the correct physical coordinates. Then in the perturbation theory we find two classes of metric perturbations, (i) stationary perturbations in a 3-dimensional vicinity of the central black hole and (ii) non-stationary perturbations in an infinitely thin disk. Since the metric perturbations directly give the matter density the theory explains the structure of disk galaxies with a bulge. As the inverse of the standard procedure the theory predicts the matter distribution for a given rotation curve. For small distances the rotation curve is Keplerian, so that the usual Schwarzschild metric and the classical Solar System tests follow.
\end{abstract}

Keywords: galactic general relativity, dark matter.

\section{Introduction}

The existence of dark matter is one of the biggest open problems in physics. Until now all searches with sensitive underground particle detectors give zero results. Then the dark matter hypothesis becomes more and more questionable. There exist various attempts of theories without dark matter, like MOND and even modified gravity theories. Contrary to these we are going to propose a much more conservative solution. We have no doubt in the validity of general relativity, because it is extremely well founded theoretically [1] and experimentally. But some new concepts are needed, both physically and mathematically.

In 2005 a conference with title "Island Universes, structure and evolution of disk galaxies" has taken place in The Netherlands [2]. This notion was really prophetic because in this article we shall apply the same mathematical technique which has been developed for cosmology in a series of papers [3-5], to now describe the gravitational field in disk galaxies. The general idea goes back to the german philosopher Immanuel Kant. In his book "Allgemeine Naturgeschichte und Theorie des Himmels" of 1755 he has described the bound systems of fixed stars as "Welten und Weltordnungen". The english name "island universes" is more appropriate. It was introduced by H.D.Curtis in 1920 in the so-called great debate over the size of the Universe Since clear observational evidence was lacking, Curtis has spoken of "the island universe theory". Now 100 years later we are going to give the theory a new meaning.

The technique goes as follows. To have more predictive power than standard theory, we start in zeroth approximation from a vacuum solution of Einstein's equation which is in agreement with the most important measurable quantities, the Hubble diagram in cosmology and the rotation curve in case of a galaxy. Then first order perturbation theory of this vacuum background leads to a finite mass content. In cosmology this gives anisotropic dust and the isotropic cosmic microwave background. In the galactic case we shall find a stationary bulge and a non-stationary infinitely thin disk. Even the satellite galaxies in planes perpendicular to the disk are given by the perturbative method. This shows that the inverse procedure of using the rotation curve to derive the matter distribution works well. At large distances where the rotation curve differs from Kepler, Newtonian gravity definitely breaks down. Then Einstein's theory has to be used, irrespective of the velocities of the stars. Astronomers thinking in terms of Newton's theory miss the point that Einstein's equations have solutions without material sources which describe very physical gravitational fields. What has been done with Newton's theory and dark matter can be done in Einstein's theory without dark matter and then even with more predictive 
power. Of course general relativity can only give the gravitational skeleton of a galaxy, the flesh (for example the thickness of the disk) must come from other physical processes.

The paper is organized as follows. In the next section we introduce the vacuum background and determine its rotation curve. This is already contained in ref.[1] and included here for completeness. In sect. 3 we calculate the metric perturbations by solving the perturbed Einstein's equations $\delta G_{\mu \nu}=8 \pi \delta t_{\mu \nu}$. Since we perturb the vacuum, the perturbed energy-momentum tensor $\delta t_{\mu \nu}$ is diagonal. Then putting the non-diagonal elements of $\delta G_{\mu \nu}=0$, we obtain the two classes of perturbations. (i) Stationary perturbations in a 3-dimensional vicinity of the central black hole and (ii) nonstationary perturbations in an infinitely thin disk. This is the gravitational skeleton of a disk galaxy with a bulge (i) and disk in the galactic plane. Obviously the infinitely thin disk gets broadened by various other processes. In sect.4 we discuss the corresponding matter density. Our conclusions are summarized in the last section.

\section{Vacuum Background and Its Rotation Curve}

The spherically symmetric cosmological background in [3-5] is given by the inner Schwarzschild solution in suitable coordinates. Then we expect that the galactic background should be the outer Schwarzschild solution in suitable coordinates. It is a general belief that coordinates are irrelevant in general relativity. As far as mathematics is concerned this is true. However for the physics the right choice of coordinates is terribly important. There is essentially only one correct set of coordinates, and which one that is, is dictated by observations. For example, the astronomer can measure the radial distance $r$ of a galaxy from the earth. He then uses this measurement to determine the rotation curve of the galaxy. But with a transformation $r^{\prime}=f(r)$ one would lose the contact with the physical reality. After this warning we write down the outer Schwarzschild metric in a slightly more general form

$$
d s^{2}=g_{\mu \nu} d x^{\mu} d x^{\nu}=e^{a} d t^{2}-e^{b} d r^{2}-r^{2} e^{c}\left(d \vartheta^{2}+\sin ^{2} \vartheta d \phi^{2}\right)
$$

where $a(r), b(r)$ and $c(r)$ are functions of $r$ only. The presence of $c(r) \not \equiv 0$ is responsible for a nontrivial rotation curve in the following. We assume that the astronomers correct their measurements for the motion of the earth with respect to the center of the galaxy, so that we can choose the center of the galaxy as origin of our coordinate system and the $z$-axis perpendicular to the galactic plane.

The covariant metric tensor corresponding to $(2.1)$ is equal to

$$
g^{\mu \nu}=\operatorname{diag}\left(e^{-a},-e^{-b},-e^{-c} / r^{2},-e^{-c} /\left(r^{2} \sin ^{2} \vartheta\right)\right)
$$

and the nonzero Christoffel symbols are given by

$$
\begin{gathered}
\Gamma_{10}^{0}=\frac{a^{\prime}}{2}, \quad \Gamma_{00}^{1}=\frac{a^{\prime}}{2} e^{a-b} \\
\Gamma_{11}^{1}=\frac{b^{\prime}}{2}, \quad \Gamma_{22}^{1}=-\frac{r^{2} c^{\prime}+2 r}{2} e^{c-b} \\
\Gamma_{33}^{1}=-\frac{r^{2} c^{\prime}+2 r}{2} e^{c-b} \sin ^{2} \vartheta \\
\Gamma_{12}^{2}=\frac{c^{\prime}}{2}+\frac{1}{r}, \quad \Gamma_{33}^{2}=-\sin \vartheta \cos \vartheta \\
\Gamma_{13}^{3}=\frac{c^{\prime}}{2}+\frac{1}{r}, \quad \Gamma_{23}^{3}=\cot \vartheta .
\end{gathered}
$$

To derive the rotation speed we must solve the geodesic equation

$$
\frac{d^{2} x^{\mu}}{d \tau^{2}}+\Gamma_{\alpha \beta}^{\mu} \frac{d x^{\alpha}}{d \tau} \frac{d x^{\beta}}{d \tau}=0
$$

where $\tau$ is an affine parameter. For motion in the galactic plane $\vartheta=\pi / 2$ we have the following three differential equations:

$$
\frac{d^{2} t}{d \tau^{2}}+a^{\prime} \frac{d t}{d \tau} \frac{d r}{d \tau}=0
$$




$$
\begin{gathered}
\frac{d^{2} r}{d \tau^{2}}+\frac{a^{\prime}}{2} e^{a-b}\left(\frac{d t}{d \tau}\right)^{2}+\frac{b^{\prime}}{2}\left(\frac{d r}{d \tau}\right)^{2}-r e^{c-b}\left(\frac{r}{2} c^{\prime}+1\right)\left(\frac{d \phi}{d \tau}\right)^{2}=0 \\
\frac{d^{2} \phi}{d \tau^{2}}+\left(\frac{2}{r}+c^{\prime}\right) \frac{d r}{d \tau} \frac{d \phi}{d \tau}=0
\end{gathered}
$$

We want to find integrating factors for these three equations. Indeed multiplying (2.5) by $\exp a$ we get

$$
\frac{d}{d \tau}\left(e^{a} \frac{d t}{d \tau}\right)=0
$$

so that

$$
e^{a} \frac{d t}{d \tau}=\text { const. }=A
$$

and

$$
\frac{d t}{d \tau}=A e^{-a} .
$$

Equation (2.7) is multiplied by $r^{2}$ which gives

$$
\frac{d}{d \tau}\left(r^{2} \frac{d \phi}{d \tau}\right)+c^{\prime} \frac{d r}{d \tau} r^{2} \frac{d \phi}{d \tau}=0 .
$$

Dividing this by $r^{2} d \phi / d \tau$ leads to

$$
\frac{d}{d \tau} \log \left(r^{2} \frac{d \phi}{d \tau}\right)+\frac{d c(r)}{d \tau}=0
$$

After integration we obtain

$$
\log \left(r^{2} \frac{d \phi}{d \tau}\right)=-c+\text { const. }
$$

so that finally

$$
\frac{d \phi}{d \tau}=\frac{J}{r^{2}} e^{-c} .
$$

Here the integration constant is chosen in such a way that $J$ reminds of the angular momentum in the standard theory. Finally we substitute (2.8) and (2.9) into (2.6). The resulting equation can be written in the form

$$
\frac{d^{2} r}{d \tau^{2}}+\frac{b^{\prime}}{2}\left(\frac{d r}{d \tau}\right)^{2}+\frac{A^{2}}{2} a^{\prime} e^{-a-b}-\frac{J^{2}}{r^{3}} e^{-b-c}\left(\frac{r}{2} c^{\prime}+1\right)=0 .
$$

Here multiplication by

$$
2 e^{b} \frac{d r}{d \tau}
$$

yields the integrable equation

$$
\frac{d}{d \tau}\left[e^{b}\left(\frac{d r}{d \tau}\right)^{2}\right]+A^{2} a^{\prime} \frac{d r}{d \tau} e^{-a}-\frac{J^{2}}{r^{3}} e^{-c} \frac{d r}{d \tau}\left(r c^{\prime}+2\right)=0 .
$$

After integration we have

$$
e^{b}\left(\frac{d r}{d \tau}\right)^{2}-A^{2} e^{-a}+\frac{J^{2}}{r^{2}} e^{-c}=\text { const. }=B .
$$

The 3 -velocity which is measured by the astronomers is equal to

$$
\vec{V}=\left(\frac{d x^{1}}{d t}, \frac{d x^{2}}{d t}, \frac{d x^{3}}{d t}\right) .
$$

Since we consider motion in the equatorial plane $\vartheta=\pi / 2$, only the first and third components are different from zero. To eliminate the affine parameter $\tau$ in favor of the measured time $t$ we multiply by appropriate powers of

$$
\frac{d \tau}{d t}=\frac{e^{a}}{A} .
$$


Then from (2.12) we get

$$
e^{b}\left(\frac{d r}{d t}\right)^{2}=e^{a}-\frac{J^{2}}{A^{2}} \frac{e^{2 a-c}}{r^{2}}+\frac{B}{A^{2}} e^{2 a} .
$$

In the following we are interested in the square

$$
\begin{aligned}
\vec{V}^{2} & =-g_{11}\left(\frac{d r}{d t}\right)^{2}-g_{33}\left(\frac{d \phi}{d t}\right)^{2}= \\
& =e^{b}\left(\frac{d r}{d t}\right)^{2}+\frac{J^{2}}{A^{2} r^{2}} e^{2 a-c} .
\end{aligned}
$$

Inserting (2.15) the term with $J^{2}$ drops out and we end up with the simple result

$$
\vec{V}^{2}=e^{a}+\frac{B}{A^{2}} e^{2 a} .
$$

The result (2.17) is not yet the desired rotation velocity because the integration constants $A$ and $B$ must still be determined. To do so we specialize everything for circular motion $r=$ const.. For $d r / d t=0$ in $(2.15)$ we get the equation

$$
\frac{J^{2}}{A^{2}} \frac{e^{-c}}{r^{2}}-e^{-a}-\frac{B}{A^{2}}=0 .
$$

A second equation is obtained by differentiating this equation with respect to $r$ which is the stability condition for the circular path:

$$
-2 \frac{J^{2}}{A^{2}} \frac{e^{-c}}{r^{3}}-\frac{J^{2}}{A^{2}} \frac{c^{\prime} e^{-c}}{r^{2}}+a^{\prime} e^{-a}=0 .
$$

This gives the following values for the integration constants

$$
\begin{gathered}
\frac{J^{2}}{A^{2}}=\frac{a^{\prime} r^{3}}{r c^{\prime}+2} e^{c-a} \\
\frac{B}{A^{2}}=\frac{r a^{\prime}}{r c^{\prime}+2} e^{-a}-e^{-a} .
\end{gathered}
$$

Here $r$ now stands for the constant radius of the circular orbit. Now we are able to compute the circular velocity squared from (2.17)

$$
\vec{V}_{c}^{2} \equiv w=\frac{r a^{\prime}}{r c^{\prime}+2} e^{a} .
$$

Next we turn to the solution of Einstein's equation for our vacuum background. From the Christoffel symbols (2.3) we can calculate the Ricci tensor. The non-vanishing components are the diagonal elements

$$
\begin{gathered}
R_{t t}=\frac{1}{2} e^{a-b}\left(a^{\prime \prime}+\frac{1}{2} a^{2}-\frac{1}{2} a^{\prime} b^{\prime}+a^{\prime} c^{\prime}+\frac{2}{r} a^{\prime}\right) \\
R_{r r}=-\frac{1}{2}\left(a^{\prime \prime}+2 c^{\prime \prime}\right)+\frac{b^{\prime}}{4}\left(a^{\prime}+2 c^{\prime}+\frac{4}{r}\right)-\frac{a^{\prime 2}}{4}-\frac{c^{\prime 2}}{2}-\frac{2}{r} c^{\prime} \\
R_{\vartheta \vartheta}=e^{c-b}\left[-1-\frac{r^{2}}{2} c^{\prime \prime}-r\left(2 c^{\prime}+\frac{a^{\prime}-b^{\prime}}{2}\right)-\frac{r^{2}}{4} c^{\prime}\left(a^{\prime}-b^{\prime}+2 c^{\prime}\right)\right]+1 \\
R_{\phi \phi}=\sin ^{2} \vartheta R_{\vartheta \vartheta},
\end{gathered}
$$

the prime always denotes $d / d r$. Let

$$
G_{\mu \nu}=R_{\mu \nu}-\frac{1}{2} g_{\mu \nu} R
$$

be the Einstein tensor then Einstein's equations without matter can be reduced to the following three differential equations

$$
G_{t t}=e^{a-b}\left[-c^{\prime \prime}-\frac{3}{4} c^{\prime 2}+\frac{1}{2} b^{\prime} c^{\prime}+\frac{1}{r}\left(b^{\prime}-3 c^{\prime}\right)\right]+\frac{1}{r^{2}}\left(e^{a-c}-e^{a-b}\right)=0
$$




$$
\begin{gathered}
G_{r r}=\frac{1}{2} a^{\prime} c^{\prime}+\frac{1}{r}\left(a^{\prime}+c^{\prime}\right)+\frac{c^{\prime 2}}{4}+\frac{1}{r^{2}}\left(1-e^{b-c}\right)=0 \\
G_{\vartheta \vartheta}=\frac{r^{2}}{2} e^{c-b}\left[a^{\prime \prime}+c^{\prime \prime}-\frac{1}{r}\left(b^{\prime}-a^{\prime}-2 c^{\prime}\right)+\frac{1}{2}\left(a^{\prime 2}-a^{\prime} b^{\prime}+a^{\prime} c^{\prime}-b^{\prime} c^{\prime}+c^{\prime 2}\right)\right]=0 .
\end{gathered}
$$

It is not hard to see that there are only two independent field equations. Indeed, using $(2.27) b$ can be expressed by $a$ and $c$. Eliminating $b$ in (2.26) and (2.28) there results one second order differential equation for $a$ and $c$ :

$$
c^{\prime \prime}=\frac{a^{\prime \prime}}{a^{\prime}}\left(c^{\prime}+\frac{2}{r}\right)+\frac{4}{r^{2}}+a^{\prime} c^{\prime}+\frac{c^{\prime 2}}{2}+\frac{2}{r}\left(a^{\prime}+c^{\prime}\right) .
$$

Introducing the new metric function

$$
f(r)=c(r)+2 \log \frac{r}{r_{c}}
$$

where $r_{c}$ has been included for dimensional reasons, equation (2.29) assumes the simple form

$$
\frac{f^{\prime \prime}}{f^{\prime}}-\frac{a^{\prime \prime}}{a^{\prime}}=a^{\prime}+\frac{f^{\prime}}{2}
$$

This can immediately by integrated

$$
\log \frac{f^{\prime}}{a^{\prime}}=a+\frac{f}{2}+\text { const. }
$$

On the other hand the circular velocity squared (2.22) now becomes

$$
w(r)=\frac{a^{\prime}}{f^{\prime}} e^{a} .
$$

Using this in (2.32) leads to

$$
f=-2 \log w
$$

and

$$
c=-2 \log \frac{r w}{r_{c}}
$$

where (2.30) has been used. This gives us the metric function

$$
e^{c}=-g_{\vartheta \vartheta} r^{-2}=\frac{r_{c}^{2}}{r^{2} w^{2}}
$$

To get $g_{t t}$ we return to $(2.32)$ which can be written as

$$
K_{a} a^{\prime} e^{a}=f^{\prime} e^{-f / 2} .
$$

Here $K_{a}$ is the integration constant in (2.32). From (2.22) we find

$$
a^{\prime} e^{a}=w\left(c^{\prime}+\frac{2}{r}\right)=\frac{d}{d r} e^{a} .
$$

Combining this with $(2.34)$

we arrive at

$$
c^{\prime}=-2 \frac{w^{\prime}}{w}-\frac{2}{r}
$$

$$
\frac{d}{d r} e^{a}=-2 w^{\prime}
$$

This gives

$$
g_{t t}=e^{a}=-2 w+K_{a} .
$$

Finally $g_{\vartheta \vartheta}$ or $\exp b$ follows from $(2.27)$. Solving for $\exp b$ we have

$$
e^{b}=a^{\prime} e^{c}\left(\frac{r^{2}}{2} c^{\prime}+r\right)+c^{\prime} e^{c}\left(\frac{r^{2}}{4} c^{\prime}+r\right)+e^{c} .
$$


Substituting (2.38) and (2.34) we find

$$
e^{b}=r_{c}^{2} \frac{w^{\prime 2}}{w^{3}}\left(\frac{1}{w}-\frac{1}{w-K_{a} / 2}\right) .
$$

We choose the integration constants $K_{a}=1$ and $r_{c}=r_{s} / 2$ where $r_{s}$ is the Schwarzschild radius

$$
r_{s}=2 M G
$$

and $M$ the mass of the central black hole. Then we get

$$
\begin{gathered}
e^{c}=\frac{r_{s}^{2}}{4 r^{2} w^{2}} \\
e^{a}=-2 w+1 \\
e^{b}=\frac{r_{s}^{2}}{4} \frac{w^{\prime 2}}{w^{4}(1-2 w)} .
\end{gathered}
$$

This is the desired background for arbitrary rotation curve. For

$$
w=\frac{r_{s}}{2 r}
$$

it reduces to the Schwarzschild solution

$$
d s^{2}=\left(1-\frac{r_{s}}{r}\right) d t^{2}-\left(1-\frac{r_{s}}{r}\right)^{-1} d r^{2}-r^{2}\left(d \vartheta^{2}+\sin ^{2} \vartheta d \phi^{2}\right) .
$$

\section{The Two Classes of Metric Perturbations}

It is our aim to solve the linear perturbation equation of Einstein's equation of the form

$$
\delta G_{\mu \nu}\left(g_{0 \alpha \beta}\right)\left(h_{\varrho \sigma}\right)=8 \pi \delta t_{\mu \nu}
$$

where $h_{\varrho \sigma}$ are the metric perturbations to be calculated. As in the cosmological calculation [4] we choose the Regge-Wheeler gauge [6] where $h_{\mu \nu}$ is of the form

$$
h_{\mu \nu}=\left(\begin{array}{cccc}
-e^{a} H_{2} & e^{b} H_{1} & 0 & 0 \\
e^{b} H_{1} & -e^{b} H_{0} & 0 & 0 \\
0 & 0 & r^{2} e^{c} K & 0 \\
0 & 0 & 0 & r^{2} e^{c} K \sin ^{2} \vartheta
\end{array}\right) Y_{l}^{m}(\vartheta, \phi) .
$$

Here $Y_{l}^{m}$ denote the spherical harmonics, the functions $H_{0}, H_{1}, H_{2}$ and $K$ depend on $t$ and $r$ only beside $l, m$. It was shown in [4] (equ.(2.13)) that the linear variation of the Einstein tensor can be obtained by covariant differentiation as follows

$$
\begin{gathered}
2 \delta G_{\mu \nu}=-\nabla^{\alpha} \nabla_{\alpha} h_{\mu \nu}+\nabla_{\nu} f_{\mu}+\nabla_{\mu} f_{\nu}-2 R_{\nu \alpha \nu}^{\beta} h_{\beta}^{\alpha}-\nabla_{\nu} \nabla_{\mu} h_{\alpha}^{\alpha}+ \\
-g_{\mu \nu}\left(\nabla^{\alpha} f_{\alpha}-\nabla^{\beta} \nabla_{\beta} h_{\alpha}^{\alpha}\right)
\end{gathered}
$$

where

$$
f_{\mu}=\nabla^{\alpha} h_{\mu \alpha} .
$$

To determine the right side of the perturbed Einstein's equation (3.1) we assume that the exact energy-momentum tensor has the perfect fluid form

$$
t_{\mu \nu}=(\varrho+p) u_{\mu} u_{\nu}-p g_{\mu \nu}
$$

where $\varrho$ and $p$ are density and pressure of the matter and $u_{\mu}$ its 4 -velocity. The vacuum as zeroth approximation is given by $\varrho_{0}=0=p_{0}$ and

$$
u_{0 \mu}=\left(e^{a / 2}, 0,0,0\right)
$$


because of the normalization $u_{\mu} u^{\mu}=1$. The first order perturbation of the vacuum is then equal to

$$
\delta t_{\mu \nu}=(\delta \varrho+\delta p) u_{0 \mu} u_{0 \nu}-\delta p g_{0 \mu \nu}
$$

where $g_{0 \mu \nu}$ is the unperturbed background metric (2.40-42). This yields the following diagonal tensor:

$$
\delta t_{\mu \nu}=\operatorname{diag}\left(e^{a} \delta \varrho, e^{b} \delta p, r^{2} e^{c} \delta p, r^{2} e^{c} \sin ^{2} \vartheta \delta p\right) .
$$

The important point is that the off-diagonal elements vanish. Then the off-diagonal components of (3.1) give linear homogeneous equations for the metric perturbations which we now study.

First we must compute

$$
f_{\mu}=g^{\nu \alpha}\left(\partial_{\alpha} h_{\mu \nu}-\Gamma_{\mu \alpha}^{\beta} h_{\beta \nu}-\Gamma_{\nu \alpha}^{\beta} h_{\mu \beta}\right)
$$

We obtain

$$
\begin{gathered}
f_{0}=\left[-\partial_{1} H_{1}-\partial_{0} H_{2}-b^{\prime} H_{1}-\left(c^{\prime}+\frac{2}{r}\right) H_{1}\right] Y \\
f_{1}=\left[e^{b-a} \partial_{0} H_{1}+\partial_{1} H_{0}+2 b^{\prime} H_{0}+\frac{a^{\prime}}{2}\left(H_{0}+H_{2}\right)+\left(c^{\prime}+\frac{2}{r}\right)\left(H_{0}-K\right)\right] Y \\
f_{2}=-K \partial_{2} Y, \quad f_{2}=-K \partial_{3} Y .
\end{gathered}
$$

We also need the background Riemann tensor, its nonzero components are equal to

$$
\begin{aligned}
& R_{101}^{0}=\frac{1}{4}\left(-2 a^{\prime \prime}-a^{\prime 2}+a^{\prime} b^{\prime}\right) \\
& R_{202}^{0}=-\frac{a^{\prime}}{4}\left(r^{2} c^{\prime}+2 r\right) e^{c-b} \\
& R_{303}^{0}=-\frac{a^{\prime}}{4}\left(r^{2} c^{\prime}+2 r\right) e^{c-b} \sin ^{2} \vartheta \\
& R_{212}^{1}=e^{c-b}\left[\left(r^{2} c^{\prime}+2 r\right)\left(\frac{b^{\prime}}{4}-\frac{c^{\prime}}{4}+\frac{1}{2 r}\right)-\frac{r \check{\mathrm{s}}}{2} c^{\prime \prime}-r c^{\prime}-1\right] \\
& R_{313}^{1}=e^{c-b}\left[\left(r^{2} c^{\prime}+2 r\right)\left(\frac{b^{\prime}}{4}-\frac{c^{\prime}}{4}+\frac{1}{2 r}\right)-\frac{r \check{s}}{2} c^{\prime \prime}-r c^{\prime}-1\right] \sin ^{2} \vartheta \\
& R_{232}^{3}=1+\frac{b^{\prime}}{2}\left(\frac{c^{\prime}}{2}+\frac{1}{r}\right) \text {. }
\end{aligned}
$$

Now the simplest computation is $\delta G_{23}$. Here only the terms

$$
-\nabla_{3} \nabla_{2} h_{\alpha}^{\alpha}=\left(-\partial_{3} \partial_{2}+\cot \vartheta \partial_{3}\right)\left(H_{0}-H_{2}-2 K\right) Y
$$

and $\nabla_{3} f_{2}, \nabla_{2} f_{3}$ contribute in (3.3), so that we obtain

$$
2 \delta G_{23}=\left(\cot \vartheta \partial_{3}-\partial_{3} \partial_{2}\right)\left(H_{0}-H_{2}\right) Y=0 .
$$

We assume $l>1$ in the following. Then this yields the first relation

$$
H_{0}(t, r)=H_{2}(t, r)
$$

which will be used at various places in order to simplify the equations.

For $\delta G_{13}$ we need

$$
\begin{aligned}
& \nabla^{\alpha} \nabla_{\alpha} h_{13}=-2 \Gamma_{\alpha 1}^{\sigma} \partial^{\alpha} h_{\sigma 3}-2 \Gamma_{\alpha 3}^{\sigma} \partial^{\alpha} h_{\sigma 1} \\
& =\left[-\left(c^{\prime}+\frac{2}{r}\right) r^{2} e^{c}\left(K+H_{0}\right) \sin ^{2} \vartheta\right] \partial^{3} Y .
\end{aligned}
$$

Here it is important to note that $h_{13}$ has to be understood as the tensor $h_{\mu \nu}$ where $\mu=1$ and $\nu=3$ is taken after applying the covariant derivatives not before (this would give $h_{13}=0$ ). The other terms which contribute are

$$
\nabla_{3} f_{1}=\partial_{3} f_{1}-\Gamma_{13}^{3} f_{3}=
$$




$$
=\left[e^{b-a} \partial_{0} H_{1}+\partial_{1} H_{0}+2\left(b^{\prime}+\frac{c^{\prime}}{2}+\frac{1}{r}\right) H_{0}+\frac{a^{\prime}}{2}\left(H_{2}-H_{0}\right)-\left(\frac{c^{\prime}}{2}+\frac{1}{r}\right) K\right] \partial_{3} Y
$$

and

This finally yields

$$
\nabla_{1} f_{3}=\partial_{1} f_{3}-\Gamma_{13}^{3} f_{3}=\left[-\partial_{1} K+\left(\frac{c^{\prime}}{2}+\frac{1}{r}\right) K\right] \partial_{3} Y
$$

$$
2 \delta G_{13}=\left[e^{b-a} \partial_{0} H_{1}+\partial_{1} H_{0}-\partial_{1} K+H_{0}\left(2 b^{\prime}-\frac{a^{\prime}}{2}\right)+H_{2} \frac{a^{\prime}}{2}-K\left(c^{\prime}+\frac{2}{r}\right)\right] \partial_{3} Y=0 .
$$

This is a first equation for the metric perturbations.

The calculation of $\delta G_{12}$ is quite similar with one very important difference. In

$$
\left.\nabla^{\alpha} \nabla_{\alpha} h_{12}=\left[-\left(c^{\prime}+\frac{2}{r}\right) r^{2} e^{c}\left(K+H_{0}\right)\right] \partial^{2} Y+\cot \vartheta\left(\frac{c^{\prime}}{2}+\frac{1}{r}\right)\left(2 K+H_{0}\right)\right] Y
$$

there is an additional term proportional to $\cot \vartheta$. It comes from

$$
g^{33} h_{22}\left(\Gamma_{33}^{2} \Gamma_{21}^{2}+\Gamma_{13}^{3} \Gamma_{33}^{2}\right)
$$

As a consequence the final result differs from (3.18)

$$
\begin{gathered}
2 \delta G_{12}=\left[e^{b-a} \partial_{0} H_{1}+\partial_{1} H_{2}-\partial_{1} K+2 b^{\prime} H_{2}-K\left(c^{\prime}+\frac{2}{r}\right)\right] \partial_{2} Y- \\
-\cot \vartheta\left(\frac{c^{\prime}}{2}+\frac{1}{r}\right)\left(H_{2}+2 K\right) Y=0 .
\end{gathered}
$$

For a nontrivial solution for the metric perturbations the last term must vanish in addition to the first. There are two possibilities for this: (i) $H_{2}=-2 K$ or (ii) $\vartheta=\pi / 2$. The first will give the bulge, the second gives the disk in the equatorial plane.

The next off-diagonal element is $\delta G_{03}$. Here we have

$$
\nabla^{\alpha} \nabla_{\alpha} h_{03}=-2 \Gamma_{33}^{1} \partial^{3} h_{01}
$$

and

$$
\nabla_{3} f_{0}=\partial_{3} f_{0}, \quad \nabla_{0} f_{3}=\partial_{0} f_{3}=-\partial_{0} K \partial_{3} Y
$$

This gives the final result

$$
2 \delta G_{03}=\left(-\partial_{0} H_{2}+\partial_{0} K-\partial_{1} H_{1}-b^{\prime} H_{1}+2 \partial_{0} K\right) \partial_{3} Y=0 .
$$

This is a second equation for the metric perturbations. Again the calculation of $\delta G_{02}$ is quite similar, but with an additional term in

Then the final result is

$$
\begin{aligned}
& \nabla^{\alpha} \nabla_{\alpha} h_{02}=-2 \Gamma_{22}^{1} \partial^{2} h_{01}+g^{33} \Gamma_{33}^{2} \Gamma_{22}^{1} h_{01}= \\
& =-\left(c^{\prime}+\frac{2}{r}\right) H_{1} \partial_{2} Y+\cot \vartheta H_{1}\left(\frac{c^{\prime}}{2}+\frac{1}{r}\right) Y .
\end{aligned}
$$

$$
2 \delta G_{02}=\left(-\partial_{0} H_{2}+\partial_{0} K-\partial_{1} H_{1}-b^{\prime} H_{1}\right) \partial_{2} Y-\cot \vartheta H_{1}\left(\frac{c^{\prime}}{2}+\frac{1}{r}\right) Y=0 .
$$

Again the last term proportional to $\cot \vartheta$ must vanish separately. Consequently in the bulge case (i) we must have $H_{1}=0$ or we are in the disk (ii) with $\vartheta=\pi / 2$.

The most complicated off-diagonal component is $\delta G_{01}$ which gives us a third equation for the metric perturbations without additional terms. The details are shown in the appendix. The final result is a second order equation:

$$
2 \delta G_{01}=\left[2 \partial_{1} \partial_{0} K+\left(2 b^{\prime}+c^{\prime}+\frac{2}{r}\right) \partial_{0} H_{2}-\left(a^{\prime}+c^{\prime}+\frac{2}{r}\right) \partial_{0} K+\right.
$$




$$
\left.+\frac{a^{\prime}-b^{\prime}}{2} \partial_{1} H_{1}+g_{1}(r) H_{1}\right] Y=0
$$

where the function $g_{1}(r)$ is given in the appendix (A.8).

The three differential equations (3.18), (3.23) and (3.26) in $(t, r)$ can be easily integrated in the bulge case (i) where

$$
H_{1}=0, \quad H_{2}=-2 K=H_{0} .
$$

Indeed equation (3.23) becomes $\partial_{0} K=0$ that means the metric perturbation is stationary. Then equ.(3.26) is identically zero, but equ.(3.18) gives

$$
3 K^{\prime}+\left(4 b^{\prime}+c^{\prime}+\frac{2}{r}\right) K=0
$$

with the solution

$$
K(r)=C_{0} r^{-2 / 3} \exp -\left(\frac{4}{3} b+\frac{c}{3}\right)
$$

where $C_{0}$ is a constant of integration. We shall discuss this further in the next section. In the disk (ii) no stationary solution is possible. Indeed, if all temporal derivatives in (3.23) and (3.26) are set $=0$, one gets a contradiction between these two equations for $H_{1}$ different from zero.

\section{Matter Density}

The matter density follows from the diagonal component

$$
\begin{gathered}
2 \delta G_{00}=-\nabla^{\alpha} \nabla_{\alpha} h_{00}+2 \nabla_{0} f_{0}-2 R_{0 \alpha 0}^{\beta} h_{\beta}^{\alpha}-\nabla_{0} \nabla_{0} h_{\alpha}^{\alpha}- \\
-g_{00}\left(\nabla^{\alpha} f_{\alpha}-\nabla^{\beta} \nabla_{\beta} h_{\alpha}^{\alpha}\right) .
\end{gathered}
$$

In calculating

$$
\begin{gathered}
\nabla^{\alpha} \nabla_{\alpha} h_{00}=g^{\mu \nu} \partial_{\mu} \partial_{\nu} h_{00}-4 \Gamma_{\alpha 0}^{\sigma} \partial^{\alpha} h_{\sigma 0}-g^{\alpha \beta} \Gamma_{\alpha \beta}^{\varrho} \partial_{\varrho} h_{00}+ \\
+2 g^{\alpha \beta} h_{\sigma 0}\left(-\partial_{\beta} \Gamma_{0 \alpha}^{\sigma}+\Gamma_{\alpha \beta}^{\varrho} \Gamma_{\varrho 0}^{\sigma}+\Gamma_{0 \beta}^{\varrho} \Gamma_{\alpha \varrho}^{\sigma}\right)+2 g^{\alpha \beta} h_{\varrho \sigma} \Gamma_{\beta 0}^{\varrho} \Gamma_{\alpha 0}^{\sigma}
\end{gathered}
$$

the angular derivatives in the first term are transformed by means of the basic relation for spherical harmonics

$$
\left(\partial_{2}^{2}+\cot \vartheta \partial_{2}+\frac{1}{\sin ^{2} \vartheta} \partial_{3}^{2}\right) Y_{l}^{m}=-l(l+1) Y_{l}^{m} .
$$

Then we obtain

$$
\begin{aligned}
\nabla^{\alpha} \nabla_{\alpha} h_{00}=- & \partial_{0}^{2} H_{2}+e^{a-b}\left[\partial_{1}^{2} H_{2}+\left(2 a^{\prime \prime}-a^{\prime 2}\right) H_{2}\right]-\frac{l(l+1)}{r^{2}} e^{a-c} H_{2}- \\
& -2 a^{\prime} \partial_{0} H_{1}+e^{a-b} \partial_{1} H_{2}\left(\frac{a^{\prime}-b^{\prime}}{2}+c^{\prime}+\frac{2}{r}\right) .
\end{aligned}
$$

Another long computation is the last term in (4.1). It is equal to

$$
\begin{gathered}
\nabla^{\alpha} f_{\alpha}-\nabla^{\beta} \nabla_{\beta} h_{\alpha}^{\alpha}=e^{-a} \partial_{0}^{2}\left(2 K-H_{2}\right)-2 e^{-a} \partial_{0} \partial_{1} H_{1}-e^{-b} \partial_{1}^{2}\left(H_{2}+2 K\right)+ \\
+\frac{l(l+1)}{r^{2}} e^{-c} K+e^{-a} \partial_{0} H_{1}\left(\frac{a^{\prime}}{2}-\frac{3}{2} b^{\prime}-2 c^{\prime}-\frac{4}{r}\right)- \\
-e^{-b} \partial_{1} H_{2}\left(\frac{3}{2} a^{\prime}+\frac{3}{2} b^{\prime}+2 c^{\prime}+\frac{4}{r}\right)+e^{-b} \partial_{1} K\left(b^{\prime}-a^{\prime}-c^{\prime}-\frac{2}{r}\right)+ \\
+e^{-b} H_{2}\left[\left(\frac{b^{\prime}-a^{\prime}}{2}-c^{\prime}-\frac{2}{r}\right)\left(a^{\prime}+2 b^{\prime}+c^{\prime}+\frac{2}{r}\right)-a^{\prime \prime}-2 b^{\prime \prime}-c^{\prime \prime}+\frac{2}{r^{2}}\right] \\
+e^{-b} K\left[-\left(\frac{b^{\prime}-a^{\prime}}{2}-c^{\prime}-\frac{2}{r}\right)\left(c^{\prime}+\frac{2}{r}\right)+c^{\prime \prime}-\frac{2}{r^{2}}\right] .
\end{gathered}
$$


There are three contributions from the Riemann tensor: $R_{010}^{1}, R_{020}^{2}$ and $R_{030}^{3}$. Putting everything together we finally get the desired matter density from

$$
\begin{gathered}
16 \pi e^{a} \delta \varrho=2 \delta G_{00}=2 e^{a-b} \partial_{1}^{2} K-e^{a-c} \frac{l(l+1)}{r^{2}} K-a^{\prime} \partial_{1} H_{1}+ \\
+\partial_{0} H_{1}\left(\frac{3}{2} a^{\prime}-\frac{b^{\prime}}{2}\right)+\partial_{1} H_{2} e^{a-b}\left(2 b^{\prime}+c^{\prime}+\frac{2}{r}\right)+e^{a-b} \partial_{1} K\left(c^{\prime}+\frac{2}{r}-b^{\prime}\right)+ \\
+H_{2} e^{a-b}\left[\frac{l(l+1)}{r^{2}}-4 a^{\prime \prime}-2 b^{\prime \prime}-c^{\prime \prime}-\frac{3}{2} a^{\prime 2}-2 a^{\prime} b^{\prime}+b^{\prime 2}-a^{\prime}\left(c^{\prime}+\frac{2}{r^{2}}\right)-\right. \\
\left.-2 a^{\prime 2}-\frac{a^{\prime} b^{\prime}}{2}+b^{\prime 2}+\left(c^{\prime}+\frac{2}{r}\right)\left(-\frac{3}{2} a^{\prime}-\frac{3}{2} b^{\prime}\right)-\left(c^{\prime}+\frac{2}{r}\right)^{2}+\frac{2}{r^{2}}\right]+ \\
+K e^{a-b}\left[\left(c^{\prime}+\frac{2}{r}\right)\left(\frac{5}{2} a^{\prime}-\frac{b^{\prime}}{2}\right)+\left(c^{\prime}+\frac{2}{2}\right)^{2}+c^{\prime \prime}-\frac{2}{r^{2}}\right] .
\end{gathered}
$$

The other diagonal components give the pressure according to (3.8), but since it is not easily measurable we do not compute it here.

To calculate the mass density profile the metric perturbations $K, H_{1}$ and $H_{2}$ are now needed. These are known in the bulge (3.29), if we know the circular velocity squared $w(r)$ in $(2.41-43)$. In the core near the central black hole we may assume the Schwarzschild value (2.44)

$$
w(r)=\frac{r_{s}}{2 r}
$$

because it is used if one determines the mass of the black hole. Then from (3.29) and (2.41-43) we find

$$
K=C_{1} \frac{w^{6}}{w^{\prime 8 / 3}}(1-2 w)^{4 / 3}=C_{2} r^{-2 / 3}\left(1-\frac{r_{s}}{r}\right)^{4 / 3}
$$

Here $C_{1}$ and $C_{2}$ are constants of integration which contain the Schwarzschild radius $r_{s}$. For the moment it is sufficient to expand the results for $r$ large compared to $r_{s}$ and calculate the leading order. Then we have $K \propto r^{-2 / 3}$ and all terms in (4.6) go with the same power of $r$, namely

$$
\delta \varrho(r) \propto r^{-8 / 3} .
$$

If one tries to test this result against observations one should keep in mind that this is the gravitational density profile. It may be changed by other physical processes.

In the disk the integration of the three evolution equations (3.18), (3.23) and (3.26) is more complicated because of the time dependence. Since the time only appears in derivatives we go over to Fourier transformed quantities

$$
\hat{K}(\omega, r)=(2 \pi)^{-1 / 2} \int K(t, r) e^{-i \omega t} d t
$$

and similarly for $H_{1}$ and $H_{2}$ and we omit the hats in the following. Then each derivative $\partial_{0}$ gives a factor $i \omega$. The three equations now become

$$
\begin{gathered}
\partial_{1} H_{1}=i \omega\left(K-H_{2}\right)-b^{\prime} H_{1} \\
\partial_{1}\left(\left(H_{2}-K\right)+2 b^{\prime} H_{2}-K\left(c^{\prime}+\frac{2}{r}\right)+i \omega e^{b-a} H_{1}=0\right. \\
2 i \omega \partial_{1} K+\left(2 b^{\prime}+c^{\prime}+\frac{2}{r}\right) i \omega H_{2}-\left(a^{\prime}+c^{\prime}+\frac{2}{r}\right) i \omega K+ \\
\frac{a^{\prime}-b^{\prime}}{2} \partial_{1} H_{1}+g_{1}(r) H_{1}=0 .
\end{gathered}
$$

To get completely real equations we divide by $i \omega$ and introduce the new metric function

$$
H_{3}(\omega, r)=\frac{H_{1}}{i \omega}
$$


Then we obtain the following first order ordinary differential equations

$$
\begin{gathered}
\partial_{1} H_{3}=K-H_{2}-b^{\prime} H_{3} \\
2 \partial_{1} K=K\left(\frac{a^{\prime}+b^{\prime}}{2}+c^{\prime}+\frac{2}{r}\right)+H_{2}\left(\frac{a^{\prime}}{2}-\frac{5}{2} b^{\prime}-c^{\prime}-\frac{2}{r}\right)+ \\
+H_{3}\left(b^{\prime} \frac{a^{\prime}-b^{\prime}}{2}-g_{1}(r)\right) \\
\partial_{1} H_{2}-\partial_{1} K=\omega^{2} H_{3} e^{b-a}-2 b^{\prime} H_{2}+K\left(c^{\prime}+\frac{2}{r}\right) .
\end{gathered}
$$

If the rotation curve in the disk is known, on can determine the metric functions $a, b$ and $c$ from (2.4143). Then these equations can be numerically integrated by any ODE-solver. This yields the metric perturbations and by (4.6) the matter density in Fourier transformed form.

\section{Conclusions}

In standard theories of galaxies one assumes some matter distribution, typically with a disk, a bulge and a halo of hypothetical dark matter. Then one calculates the rotation curves by means of Newtonian gravity, saying that the velocities of the stars are small so that the relativistic theory is not needed. However, to understand the nontrivial rotation curves there are two possibilities: Either there is dark matter and Newton is right - or Newton is wrong. Since direct searches for dark matter have been negative we advocate the second possibility. But different from the MOND paradigm we fully believe in general relativity as the correct gravity theory on all scales. After all it would be curious if this beautiful theory would only be needed to calculate small corrections to Newton apart from singular phenomena as black holes and the early universe.

Our main conclusion is that general relativity is strongly at work in galaxies. In contrast to standard theory we do not start from some matter distribution, but from the observed rotation curves. They provide the gravitational background of the galaxy and the perturbed Einstein's equations then give the matter density. In this way the structure of disk galaxies is derived from the theory, not assumed. A last highlight can be added. The Milky Way and other galaxies are surrounded by satellite galaxies [7] which are located in planes $\phi=$ const. perpendicular to the disk. Applying our perturbation theory to those planes we find non-zero solutions for the metric perturbations and, hence, for the matter density. So even this detail is correctly described by galactic general relativity. The standard dark matter theory is in difficulty here [7].

\section{References}

1. Scharf G.2016, Gauge Field Theories, Spin 1 and Spin 2, 100 Years After General Relativity, Dover Publications, Inc. Mineola, New York

2. De Jong R.S. Editor, Island Universes,Structure and Evolution of Disk Galaxies, 2007 Springer

3. Scharf G. 2018, Cosmology with the cosmic rest frame, arXiv 1503.05878

4. Scharf G. 2019, Perturbation theory in nonstandard cosmology, Adv. Astrophys. 4(4), 133, arXiv 1905.02526

5. Scharf G. 2020. Cosmology without dark matter, Adv.Astrophys. 5(1), 1

6. Regge T., Wheeler J.A. 1957, Physical Review 108, 1063

7. Müller O., Pawlowski M.S., Jerjen H., Lelli F. 2018, Science 359, 534

\section{Appendix}

Here we calculate

$$
2 \delta G_{01}=\nabla_{1} f_{0}+\nabla_{0} f_{1}-2 g^{\sigma \alpha} R_{0 \sigma 1}^{\varrho} h_{\varrho \alpha}-\nabla_{1} \nabla_{0} h_{\alpha}^{\alpha}-\nabla^{\alpha} \nabla_{\alpha} h_{01} .
$$

First we compute

$$
\nabla^{\alpha} \nabla_{\alpha} h_{01}=g^{\mu \nu} \partial_{\mu} \partial_{\nu} h_{01}-2 \Gamma_{\alpha 0}^{\sigma} \partial^{\alpha} h_{\sigma 1}-2 \Gamma_{\alpha 1}^{\sigma} \partial^{\alpha} h_{\sigma 0}+
$$




$$
+g^{\alpha \beta} h_{\sigma 1}\left(-\partial_{\beta} \Gamma_{0 \alpha}^{\sigma}+\Gamma_{\alpha \beta}^{\varrho} \Gamma_{\varrho 0}^{\sigma}+\Gamma_{0 \beta}^{\varrho} \Gamma_{\alpha \varrho}^{\sigma}\right) .
$$

The second order differential operator is equal to

$$
\begin{aligned}
& {\left[e^{-a} \partial_{0}^{2}-e^{-b} \partial_{1}^{2}-\frac{e^{-c}}{r^{2}} \partial_{\vartheta}^{2}-\frac{e^{-c}}{r^{2} \sin ^{2} \vartheta} \partial^{2} \phi\right] h_{01}=} \\
= & {\left[e^{-a} \partial_{0}^{2}-e^{-b} \partial_{1}^{2}\right] h_{01}+e^{-c} \frac{l(l+1)}{r^{2}} e^{b} H_{1} Y_{l}^{m}(\vartheta, \phi) }
\end{aligned}
$$

where we have transformed the angular derivatives by means of the identity (4.3). The term with $\cot \vartheta$ drops out so that the final result is proportional to $Y$ :

$$
\begin{gathered}
\nabla^{\alpha} \nabla_{\alpha} h_{01}=\left[\left(e^{-a} \partial_{0}^{2}-e^{-b} \partial_{1}^{2}-e^{-c} \frac{l(l+1)}{r^{2}}\right) e^{b} H_{1}+\partial_{1} H_{1}\left(\frac{a^{\prime}}{2}+\frac{3}{2} b^{\prime}-c^{\prime}-\frac{2}{r}\right)+\right. \\
\left.+2 a^{\prime} \partial_{0} H_{2}+H_{1}\left(\frac{a^{\prime \prime}+b^{\prime \prime}}{2}+a^{\prime 2}+b^{\prime 2}-a^{\prime} b^{\prime}+\frac{a^{\prime} c^{\prime}}{2}-\frac{b^{\prime} c^{\prime}}{2}+\frac{a^{\prime}-b^{\prime}}{r}\right)\right] Y .
\end{gathered}
$$

Next we compute

$$
\begin{aligned}
\nabla_{1} f_{0}= & \partial_{1} f_{0}-\Gamma_{01}^{0} f_{0}=\left[-\partial_{1} \partial_{0} H_{2}-\partial_{1}^{2} H_{1}+\partial_{1} H_{1}\left(\frac{a^{\prime}}{2}-b^{\prime}-c^{\prime}-\frac{2}{r}\right)+\right. \\
& \left.+\frac{a^{\prime}}{2} \partial_{0} H_{2}+H_{1}\left(-b^{\prime \prime}-c^{\prime \prime}+\frac{a^{\prime} b^{\prime}}{2}+\frac{a^{\prime} c^{\prime}}{2}+\frac{a^{\prime}}{r}+\frac{2}{r}\right)\right] Y
\end{aligned}
$$

and

$$
\begin{gathered}
\nabla_{0} f_{1}=\partial_{0} f_{1}-\Gamma_{01}^{0} f_{0}= \\
=\left[e^{b-a} \partial_{0}^{2} H_{1}+\partial_{0} \partial_{1} H_{0}+2 b^{\prime} \partial_{0} H_{0}+a^{\prime} \partial_{0} H_{2}+\partial_{0} H_{0}\left(\frac{a^{\prime}}{2}+c^{\prime}+\frac{2}{r}\right)+\frac{a^{\prime}}{2} \partial_{1} H_{1}-\right. \\
\left.-\left(c^{\prime}+\frac{2}{r}\right) \partial_{0} K+\frac{a^{\prime}}{2}\left(b^{\prime}+c^{\prime}+\frac{2}{r}\right) H_{1}\right] Y .
\end{gathered}
$$

From the Riemann tensor we obtain

$$
g^{11} R_{101}^{0}=e^{-b}\left(\frac{a^{\prime} b^{\prime}}{4}-\frac{a^{\prime \prime}}{2}-\frac{a^{\prime 2}}{4}\right)
$$

times $-2 h_{01}$. Finally

$$
\nabla_{1} \nabla_{0} h_{\alpha}^{\alpha}=\partial_{1} \partial_{0} h_{\alpha}^{\alpha}-\Gamma_{01}^{0} \partial_{9} h_{\alpha}^{\alpha}=-2 \partial_{1} \partial_{0} K+a^{\prime} \partial_{0} K .
$$

Inserting everything into (A.1) we get the equation (3.26) with

$$
\left.g_{1}(r)=\frac{a^{\prime \prime}-b^{\prime \prime}}{2}-c^{\prime \prime}-\frac{a^{\prime 2}}{2}+\frac{3}{2} a^{\prime} b^{\prime}+\frac{b^{\prime} c^{\prime}}{2}+\frac{a^{\prime}}{2}\left(c^{\prime}+\frac{2}{r}\right)+\frac{2}{r^{2}}-e^{b-c} \frac{l(l+1)}{r^{2}}\right] .
$$

